\title{
Search for Stable Massive Particles with the ATLAS detector in proton-proton collisions at $\sqrt{\mathrm{s}}=13 \mathrm{TeV}$
}

\author{
Sascha Mehlhase \\ On behalf of the ATLAS Collaboration \\ Ludwig-Maximilians-Universität München \\ E-mail: sascha.mehlhase@physik.uni-muenchen.de
}

\begin{abstract}
A search for heavy long-lived charged $R$-hadrons is performed using data from proton-proton collisions at $\sqrt{s}=13 \mathrm{TeV}$ collected by the ATLAS Experiment at the Large Hadron Collider at CERN. The analysis utilises both time-of-flight and specific-ionisation energy-loss measurements to derive the velocity and subsequently the mass of the particles and places upper cross-section limits in a mass range from $600 \mathrm{GeV}$ to $2000 \mathrm{GeV}$ as well as mass exclusion limits for stable $R$-hadrons.
\end{abstract}

38th International Conference on High Energy Physics

3-10 August 2016

Chicago, USA 


\section{Introduction}

Heavy long-lived particles (LLP), observable at the Large Hadron Collider (LHC), are predicted in a variety of theories extending the Standard Model (SM), with supersymmetry (SUSY) [1-6] just being one example. Supersymmetry allows for long-lived charged particles - sleptons $(\tilde{\ell})$, squarks $(\tilde{q})$, gluinos $(\tilde{g})$ and charginos $\left(\tilde{\chi}_{1}^{ \pm}\right)$- in models that either violate [7-9] or conserve [10$16] R$-parity. In this contribution a first search for composite colourless states of squarks or gluinos together with SM quarks or gluons, called $R$-hadrons [10], stable enough to reach and leave the detector, using $3.2 \mathrm{fb}^{-1}$ of proton-proton collisions at $\sqrt{s}=13 \mathrm{TeV}$ collected by the ATLAS experiment, is sketched (see Reference [17] for details). With an expected propagation velocity, $\beta=v / c$, substantially lower than one and a specific ionisation energy loss, $\mathrm{d} E / \mathrm{d} x$, larger than that for any charged SM particle, the search takes advantage of both discriminants to identify these LLPs within the ATLAS experiment.

\section{ATLAS detector and observables}

The ATLAS detector [18] is a multi-purpose particle-physics detector consisting of an inner detector (ID) immersed in an axial magnetic field to reconstruct trajectories of charged particles, calorimeters to measure the energy of particles that interact electromagnetically or hadronically and a muon spectrometer within a toroidal magnetic field to provide tracking for muons. With near $4 \pi$ coverage in solid angle, the ATLAS detector is able to deduce the missing transverse momentum, $\vec{p}_{\mathrm{T}}^{\text {miss }}$, associated with each event. In particular the innermost silicon pixel detector allows for a $\beta \gamma /$ mass estimate using $\mathrm{d} E / \mathrm{d} x$ and momentum ( $p$ ) measurements fitted to an empirical BetheBloch function (calibrated using low-momentum pions, kaons and protons), whereas the hadronic tile-calorimeter allows for a $\beta$ / mass estimate using time-of-flight measurements in cells crossed by extrapolated candidate tracks (calibrated using $Z \rightarrow \mu \mu$ events in data and simulation).

\section{Data and simulated samples}

The search presented in this contribution is based on $3.2 \mathrm{fb}^{-1}$ of $p p$ collision data collected in 2015 at a centre-of-mass energy $\sqrt{s}=13 \mathrm{TeV}$. Reconstructed $Z \rightarrow \mu \mu$ events in data and simulation are used for timing resolution studies. Signal events - simulated in PYTHIA 6.427 [19] with the AUET2B [20] set of tuned parameters for the underlying event and the CTEQ6L1 [21] parton distribution function (PDF) set, incorporating PYTHIA-specific specialised hadronisation routines [22-24] - are used to study the expected signal behaviour. Sparticle-sparticle events simulated in MG5_aMC@NLO v2.2.3.p0 [25] are used to reweight the PYTHIA samples to a more realistic description of $\mathrm{QCD}$ radiative effects.

\section{Event and candidate selection}

Events are required to pass a $70 \mathrm{GeV}$ missing-transverse-momentum trigger-threshold, have all relevant detector components fully operational, a primary vertex (PV) built from at least two wellreconstructed charged-particle tracks $\left(p_{\mathrm{T}}>400 \mathrm{MeV}\right)$, and at least one $R$-hadron candidate track. Initial candidates are based on central, high- $p_{\mathrm{T}}$ inner detector tracks, with quality requirements ensuring good $p, \mathrm{~d} E / \mathrm{d} x$ and $\beta$ measurements, and are also used as basis for background estimate. Signal candidates are required to have $p>200 \mathrm{GeV}, \beta \gamma<1.35(<1.15)$ for $R$-hadron masses up to (greater than) $1.4 \mathrm{TeV}$ and $\beta<0.75$. The mass-hypothesis-dependent signal regions are defined by increasing requirements on $m_{\beta \gamma}$ and $m_{\beta}$ (about $2 \sigma$ below nominal $R$-hadron mass, given the expected resolution). 

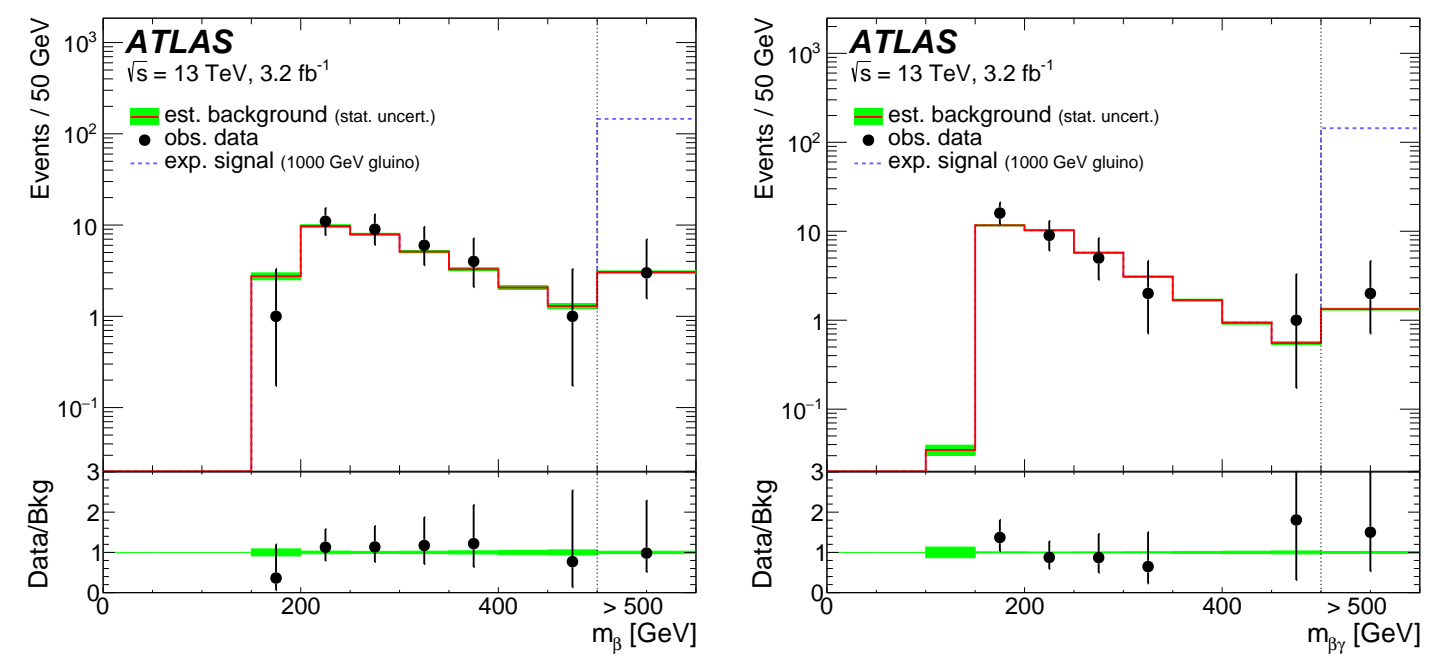

Figure 1: Data (black dots) and background estimates (red solid line) for $m_{\beta}$ (left) and $m_{\beta \gamma}$ (right) for the gluino $R$-hadron search $(1000 \mathrm{GeV})$. The green shaded band illustrates the statistical uncertainty of the background estimate. The blue dashed lines illustrate the expected signal (on top of background) for the given $R$-hadron mass hypothesis. The black dashed vertical lines at $500 \mathrm{GeV}$ show the mass selection and the last bin includes all entries/masses above. [17]

\section{Background estimation}

The background is estimated in a data-driven way. First, probability distribution functions (pdf) as a function of the momentum, $\beta$ and $\beta \gamma$ values, are determined from data. These pdfs are produced from candidates in data, which have passed the initial selection, but fall in sidebands of the signal region. Background distributions as a function of $m_{\beta}$ and $m_{\beta \gamma}$ are obtained by randomly sampling these pdfs and using the equation $m={ }^{p} / \beta \gamma$. Each $R$-hadron mass hypothesis has a different selection, and therefore corresponding individual background estimates are produced accordingly. The mass distributions, normalised to data events outside the signal region (i.e. not passing both mass requirements of the hypothesis in question), are shown in Figure 1 along with the data and the expected signal for the $1000 \mathrm{GeV}$ gluino $R$-hadron mass hypothesis.

\section{Systematic uncertainties}

Systematic uncertainties are derived using data, whenever possible. This is not the case for theoretical cross sections (14\% - 57\%, increasing with mass hypothesis) and ISR effects, where the latter are folded with the trigger efficiency curve obtained from data to produce the overall $E_{\mathrm{T}}^{\text {miss }}$ trigger efficiency. The overall uncertainties on signal efficiency, background estimate and luminosity are estimated to be $20 \%-16 \%$ (decreasing with mass hypothesis), $30 \%-43 \%$ (increasing with mass hypothesis) and 5\%, respectively.

\section{Results}

Mass distributions of events for a $1000 \mathrm{GeV}$ gluino $R$-hadron mass hypothesis are shown in Figure 2 (left). At no point in the examined mass range does this search exhibit any statistically significant excess of events above the expected background, which is $1.23 \pm 0.37$ for a $1000 \mathrm{GeV}$ and $0.185 \pm$ 0.071 for a $1600 \mathrm{GeV}$ mass hypotheses, respectively. Therefore, 95\% CL upper limits are placed 

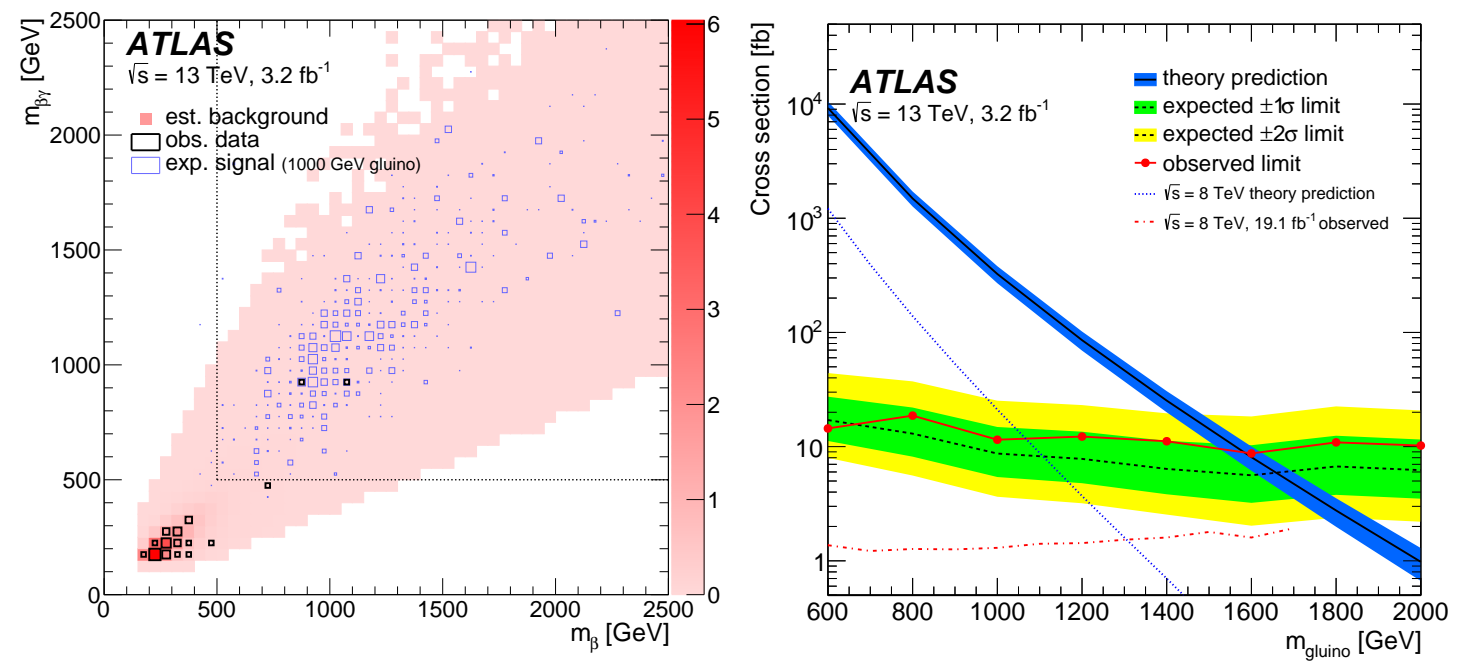

Figure 2: left: Data (bold boxes) and background estimates (colour fill) for $m_{\beta}$ vs. $m_{\beta \gamma}$ for the gluino $R$-hadron search $(1000 \mathrm{GeV})$. The blue thin-line boxes illustrate the expected signal (on top of background) for the given $R$-hadron mass hypothesis. The black dashed vertical/horizontal lines at $500 \mathrm{GeV}$ show the mass selection (signal region in the top-right). Two events pass this selection. [17]

right: Expected (dashed black line) and observed (solid red line) 95\% CL upper limits on the cross section as a function of mass for the production of long-lived gluino $R$-hadrons. The theory prediction along with its $\pm 1 \sigma$ uncertainty is show as a black line and a blue band, respectively. The observed 8 TeV Run-1 limit and theory prediction [27] are shown in dash-dotted and dotted lines, respectively. [17]

on the $R$-hadron production cross section, with an example shown in Figure 2 (right). Long-lived $R$-hadrons containing a gluino, bottom or top squark are excluded at $95 \% \mathrm{CL}$ for masses up to $1580 \mathrm{GeV}, 805 \mathrm{GeV}$ and $890 \mathrm{GeV}$, respectively [17]. These limits are derived from the expected signal and the estimated background in the signal region and using a one-bin counting experiment applying the $C L_{S}$ prescription [26], and substantially extend previous ATLAS and CMS limits from 8 TeV Run-1 data in case of gluino $R$-hadrons and are complementary to searches for SUSY particles which decay promptly.

\section{References}

[1] Yu.A. Gol'fand, E.P. Likhtman; JETP Lett. 13 (1971) 323

[2] D.V. Volkov, V.P. Akulov; Phys. Lett. B 46 (1973) 109

[3] J. Wess, B. Zumino; Nucl. Phys. B 70 (1974) 39

[4] J. Wess, B. Zumino; Nucl. Phys. B 78 (1974) 1

[5] S. Ferrara, B. Zumino; Nucl. Phys. B 79 (1974) 413

[6] A. Salam, J.A. Strathdee; Phys. Lett. B 51 (1974) 353

[7] H.K. Dreiner; Adv. Ser. Direct. High Energy Phys. 21 (1997) 565

[8] E.L. Berger, et al.; Phys. Rev. Lett. 92 (2004) 201801

[9] R. Barbier, et al.; Phys. Rept. 420 (2005) 1

[10] G.R. Farrar, P. Fayet; Phys. Lett. B 76 (1978) 575

[11] M. Fairbairn, et al.; Phys. Rept. 438 (2007) 1

[12] C.F. Kolda; Nucl. Phys. Proc. Suppl. 62 (1998) 266

[13] H. Baer, et al.; Phys. Rev. D 59 (1999) 075002

[14] S.J. Gates Jr., O. Lebedev; Phys. Lett. B 477 (2000) 216
[15] G.F. Giudice, A. Romanino; Nucl. Phys. B 699 (2004) 65

[16] N. Arkani-Hamed, et al.; Nucl. Phys. B 709 (2005) 3

[17] ATLAS Collaboration; Phys. Lett. B 760 (2016) 647

[18] ATLAS Collaboration; JINST 3 (2008) S08003

[19] T. Sjöstrand, et al.; JHEP 05 (2006) 26

[20] ATLAS Collaboration; ATL-PHYS-PUB-2011-014 (2011)

[21] J. Pumplin, et al.; JHEP 07 (2002) 12

[22] T. Sjöstrand, et al; http://home.thep.lu.se/torbjorn/pythiaaux/past.html

[23] A.C. Kraan; Eur. Phys. J. C 37 (2004) 91

[24] G.R. Farrar, et al.; JHEP 02 (2011) 18

[25] J. Alwall, et al.; JHEP 07 (2014) 79

[26] A.L. Read; J. Phys. G 28 (2002) 2693

[27] ATLAS Collaboration; JHEP 01 (2015) 68 